\title{
BMJ Open Access to infertility evaluation and treatment in two public fertility clinics and the reasons for withholding it: a prospective survey cohort study of healthcare professionals
}

\author{
Evangelia Elenis (D) , ${ }^{1}$ Agneta Skoog Svanberg, ${ }^{1}$ Pia Leandersson, ${ }^{2}$ Judith Lind, ${ }^{3}$ \\ Gunilla Sydsjö ${ }^{4}$
}

To cite: Elenis E, Skoog Svanberg A, Leandersson P, et al. Access to infertility evaluation and treatment in two public fertility clinics and the reasons for withholding it: a prospective survey cohort study of healthcare professionals. BMJ Open 2020;10:e041538. doi:10.1136/ bmjopen-2020-041538

- Prepublication history for this paper is available online To view these files, please visit the journal online (http://dx.doi. org/10.1136/bmjopen-2020041538).

Received 10 June 2020 Revised 23 November 2020 Accepted 26 November 2020

Check for updates

(C) Author(s) (or their employer(s)) 2020. Re-use permitted under CC BY-NC. No commercial re-use. See rights and permissions. Published by BMJ.

For numbered affiliations see end of article.

Correspondence to Dr Evangelia Elenis; evangelia.elenis@kbh.uu.se

\section{ABSTRACT}

Objectives Study the proportion of patients affected by involuntary childlessness who are denied fertility treatment and the reasons behind this in a publicly funded healthcare system.

Design Survey study using prospectively collected information by healthcare professionals.

Setting Two university-affiliated fertility clinics in Sweden. Participants Single women and couples in heterosexual and homosexual relationships seeking fertility evaluation and treatment between November 2017 and April 2018 (943 individual cases).

Primary and secondary outcome measures Number and proportion of individuals who were either denied, delayed or granted fertility treatment directly. Furthermore, the reasons behind delaying or completely withholding treatment.

Results The majority of those seeking evaluation were heterosexual couples (75\%), while $14 \%$ were single women and $7.5 \%$ were same-sex couples. The great majority of those undergoing evaluation were granted treatment either directly $(85 \%)$ or after in-depth evaluation (7.5\%), while $7.5 \%$ were denied treatment. Among those who were denied treatment, there were a greater proportion of single women and couples seeking treatment with donated gametes. Among heterosexual couples, gamete origin was not associated with treatment refusal. Although age did not differ between those granted and denied treatment, a higher body mass index (in both recipient and partner, when applicable) was observed among those being refused treatment. Fertility specialists in Sweden focused their assessment on parental factors that may indirectly entail a risk of harm to the future child, such as medical and psychiatric conditions of the individuals involved, their financial constraints and other social reasons, substance abuse and female obesity. Conclusion Being single or receiving treatment with donated gametes can both be reasons for withholding fertility treatment. Although difficult to operationalise, parenting assessment in Sweden is employed interchangeably in treatments with donated gametes (legally mandated assessment) and even autologous gametes (non-legally mandated assessment)—making
Strengths and limitations of this study

- This is the largest study on the subject including both publicly subsidised and self-funded in vitro fertilisation treatments performed with both autologous and donated gametes.

- The survey protocol was constructed based on the recommendations concerning the evaluation of parental suitability issued by a national authority, the Swedish Board of Health and Welfare.

- It is uncertain what the study findings would be if the treatment cycles were entirely self-funded, and not largely publicly financed as it is in the Swedish healthcare system.

evident a need for clear official policy guidelines regulating these assessments and the provision of treatment.

\section{INTRODUCTION}

Access to treatment with assisted reproductive technologies (ART) is most often restricted by the implementation of eligibility criteria imposed by national ART policies concerning the welfare of the individuals involved. Among the common reasons for withholding fertility treatment are reported prior medical and psychiatric conditions; ${ }^{1}$ inadequate financial resources; ${ }^{2}$ previous convictions $^{3}{ }^{4}$; child protection orders $;^{4}$ drug or alcohol abuse; ${ }^{5}$ current tobacco use; ${ }^{6}$ and obesity. ${ }^{6} 7$ Many of these criteria are justified with reference to the health of the intended mother or the welfare of the child. Services may be withheld if a pregnancy entails risks to the mother or if there is a risk that diseases may be transmitted to the child through conception, which might jeopardise the life or health of the child. ${ }^{8}$ Furthermore, in many countries, restrictions to access to ART are justified with reference 
to the candidates' assumed suitability as parents and thus to the child's welfare and upbringing. Until recently, the child's claimed need for a father excluded lesbian couples, as well as single women, from gaining access to ART treatment in many countries. ${ }^{9} 10$ The degree of refusal for treatment, as well as the circumstances under which ART is regulated and used, has not, however, been fully explored in the literature. Previous research on the regulation of access to ART services at clinical level is mainly retrospective and based on surveys ${ }^{11}$ or interviews with clinic staff. ${ }^{12}$ Lee et $a l^{13}$ reported that out of $20 \mathrm{UK}$ clinics, 15 reported refusing treatment in up to 10 cases of concern for the welfare of the child in a year. A questionnaire study with 210 directors of ART programmes in the USA showed that, on average, programmes reported denying treatment to $4 \%$ of their candidates each year: $3 \%$ for medical reasons and $1 \%$ for social and psychological reasons. ${ }^{14}$ A general conclusion of these studies is that clinics' assessment and regulation practices vary considerably.

Swedish legislation requires that all individuals and couples receiving treatment with donated gametes must undergo a psychological evaluation in order to assess their parenting adequacy. ${ }^{815}$ This should include assessment of the stability of the relationship between the prospective parents, their psychosocial and financial circumstances and their social networks, and should determine if there is any history of violence. ${ }^{8}$ The clinicians are prompted to consult with experts, such as psychiatrists and psychologists, or even to refer candidates for professional in-depth psychosocial evaluation. It is recommended that a professional with competencies in the behavioural sciences participate in the assessment. ${ }^{8}$ Furthermore, since treatment with donated gametes is non-anonymous in Sweden, the intended parents are assessed regarding their willingness to disclose the manner of conception to their offspring. In contrast to practice in other countries such as the UK, Swedish ART policies prescribe that an assessment should be made of the candidates' psychological and social circumstances in order to ensure that the child 'will grow up in good circumstances', only when ART treatment involves gametes from a donor. ${ }^{15} 16$ However, there is no corresponding requirement when fertility treatments are performed with autologous gametes. When considering the candidacy of single individuals for ART, an additional assessment is made regarding whether they have a robust social profile and whether they can rely on supportive family and social network for the upbringing of the child. ${ }^{17}$

In addition to the above eligibility criteria, the Swedish Association of Local Authorities and Regions issued further eligibility criteria for publicly subsidised treatment ${ }^{18}$ which include childlessness, and age below 40 years for the woman undergoing treatment and below 56 years for her partner. Individual clinics may have additional eligibility criteria concerning body mass index (BMI) and the absence of psychological and social contraindications to pregnancy and parenthood.
Because the Swedish healthcare system is a publicly funded system, it is reasonable to assume that there is less self-exclusion of candidates because of financial constraints. It may also be assumed that the proportion of socioeconomically challenged candidates among fertility treated individuals in Sweden may be higher than in countries with less generous public healthcare schemes. The aim of the present study was therefore to explore the proportion of candidates who are denied treatment among those who seek ART treatment at a public healthcare system, such as that of Sweden. The secondary aim was to examine the reasons for treatment being withheld.

\section{MATERIALS AND METHODS \\ Study protocol}

The current study is a prospective observational cohort study that was conducted as a quantitative study at two university-affiliated fertility clinics in Sweden. Both clinics performed mainly publicly funded as well as a small number of self-funded ART treatments. Data on individuals seeking fertility assistance were collected with the aid of a study-specific survey protocol. The content of the protocol was based on the guidelines for the psychosocial assessment of candidates for donor ART treatment issued by the Swedish National Board of Health and Welfare (NBHW) in 2016. ${ }^{17}$ The assessment areas listed in these guidelines include age, health and disabilities, social and financial circumstances, couple relationship and social networks (particularly emphasised in relation to single candidates). Members of staff at each clinic were instructed to fill out one protocol for all consecutive patients seeking fertility evaluation and/or treatment over a period of 6 months (November 2017 through April 2018). Cases refer to either single individuals or infertile couples contacting the fertility clinic directly or after referral by other healthcare specialists. Candidates included heterosexual couples who intended to receive treatment with their own gametes, heterosexual couples who intended to receive treatment with oocyte donation, as well as heterosexual and female same-sex couples or single women who intended to receive treatment with donated sperm. The medical reasons for why heterosexual couples were considered for oocyte or sperm donation were not collected in the study protocol. Embryo donation and simultaneous donation of both egg and sperm were not permitted in Sweden during the study period. The information on which the assessment of eligibility for treatment was based was collected by healthcare professionals through self-report by the treatment candidates, as well as by scrutinising the candidates' medical records.

\section{Study population}

In this study, a total of 943 cases were included during the study period; among those, $704(74.7 \%)$ cases concerned heterosexual couples, $71(7.5 \%)$ concerned same-sex (female) couples, $136(14.4 \%)$ were single women and, in $32(3.4 \%)$ instances, information regarding the 


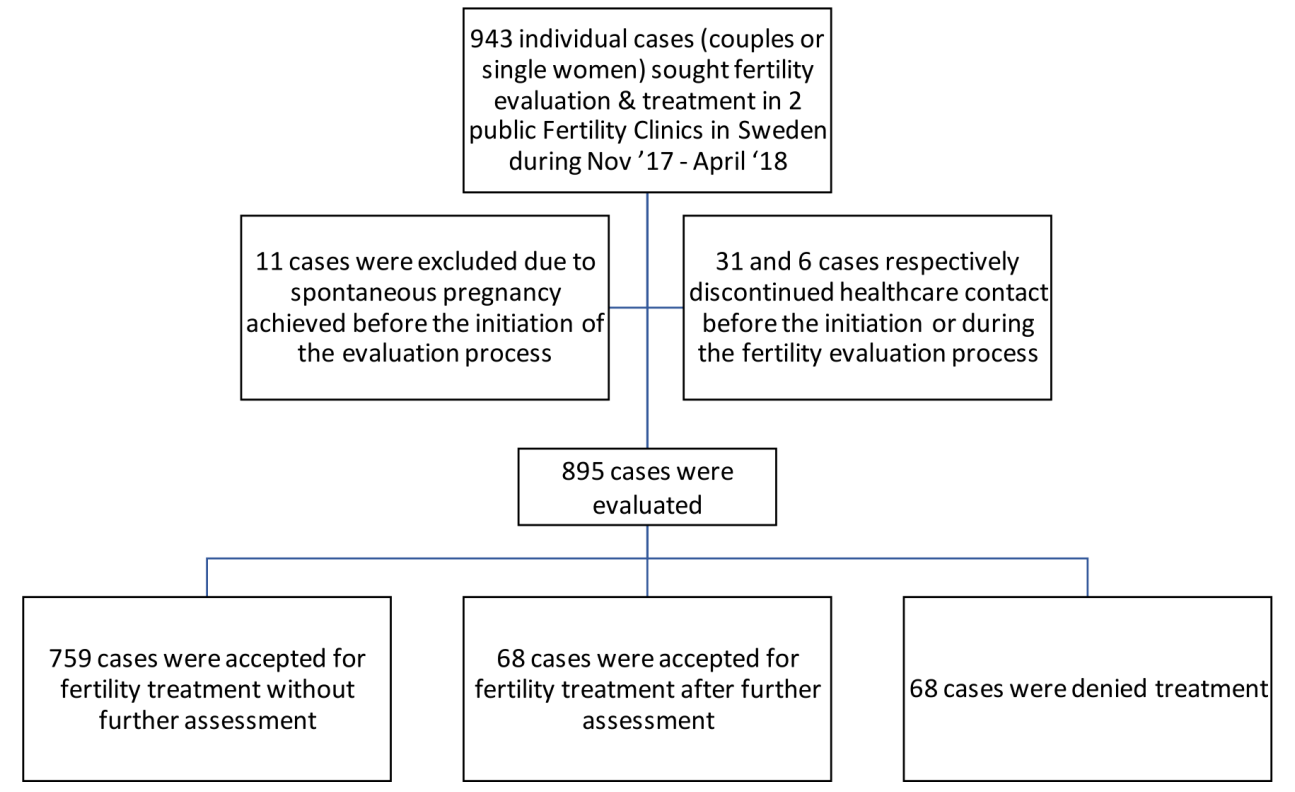

Figure 1 Flow chart of study participants.

relationship status was missing. Thirty-one cases discontinued the evaluation process of their own accord before the initiation and six during the screening process. The reasons behind the voluntary discontinuation remain unknown. Eleven additional cases refrained from undergoing treatment due to achieving spontaneous conception before the initiation of the fertility assessment (figure 1). Ultimately, 895 cases completed the evaluation process.

\section{Patient and public involvement}

There was no patient or public involvement in the design, conduct, reporting or dissemination plans of our research study.

\section{Statistical analyses}

All analyses in the current study were performed using the Statistical Package for the Social Sciences (SPSS) V.2 (IBM). Data for categorical variables are presented as numbers (n) and per cent (\%), while for continuous variables mean and SD are used. Differences across groups (composition of the couple and whether accepted for treatment or not) with respect to continuous variables (BMI and age) were analysed using analysis of variance, followed by individual group comparisons with Student's t-test. Tukey's adjustment for multiple comparisons was applied in these comparisons. Pearson's $\mathrm{X}^{2}$ test was used to analyse the relationship between categorical variables. A pvalue $<0.05$ (two sided) was considered statistically significant.

\section{RESULTS}

Single women seeking fertility treatment had a higher mean age compared with women in both same-sex $(p<0.001)$ and heterosexual relationships $(p<0.001)$. However, the recipients' BMI was comparable among groups $(\mathrm{p}=0.610)$. No substantial variability between centres could be seen (data not shown). The partner's age was higher among heterosexual couples than among same-sex couples $(p<0.001)$. There was a greater proportion of heterosexual couples compared with same-sex (female) couples seeking public treatment $(86.9 \%$ vs $75.4 \%$, respectively, $\mathrm{p}<0.05$ ) compared with self-funded.

Among participants who completed the entire fertility assessment process $(\mathrm{n}=895), 68$ cases $(7.6 \%)$ were denied treatment, $68(7.6 \%)$ received treatment after further in-depth evaluation and 759 (84.8\%) were accepted for treatment directly (table 1 ). A significantly higher proportion of candidates seeking treatment with donated gametes (both public and private treatments) were denied treatment (11.7\%) compared with $4.8 \%$ of those seeking treatment with autologous gametes. The recipient's age did not differ across the various outcome categories (ie, granted/delayed/withheld treatment), nor did partner's age when comparing those granted treatment to those with a delayed treatment. However, a higher (mean) recipient's BMI as well as a higher partner's BMI was observed among those who were delayed or denied treatment compared with individuals granted treatment. Furthermore, a higher proportion of single women $(15.9 \%)$ were denied treatment compared with couples $(4.8 \%$ among heterosexual couples and $7.1 \%$ among same-sex couples $)(p<0.001)$. Among heterosexual couples, the reported rates of withheld treatment were similar both in donated and autologous gamete cycles $(5.9 \%$ vs $4.8 \%$, respectively) ( $\mathrm{p}=0.832)$ (table 2 ).

Women with BMI exceeding the limit were placed on active review (17.2\%) and were accepted for treatment when the BMI fell within the acceptable range set by each clinic. Individuals with psychiatric or physical conditions were accepted for treatment after in-depth evaluation and after contacting the physician treating them for 
Table 1 Background information of study participants in relation to the outcome (granted/delayed/withheld ART treatment) $(n=895)$

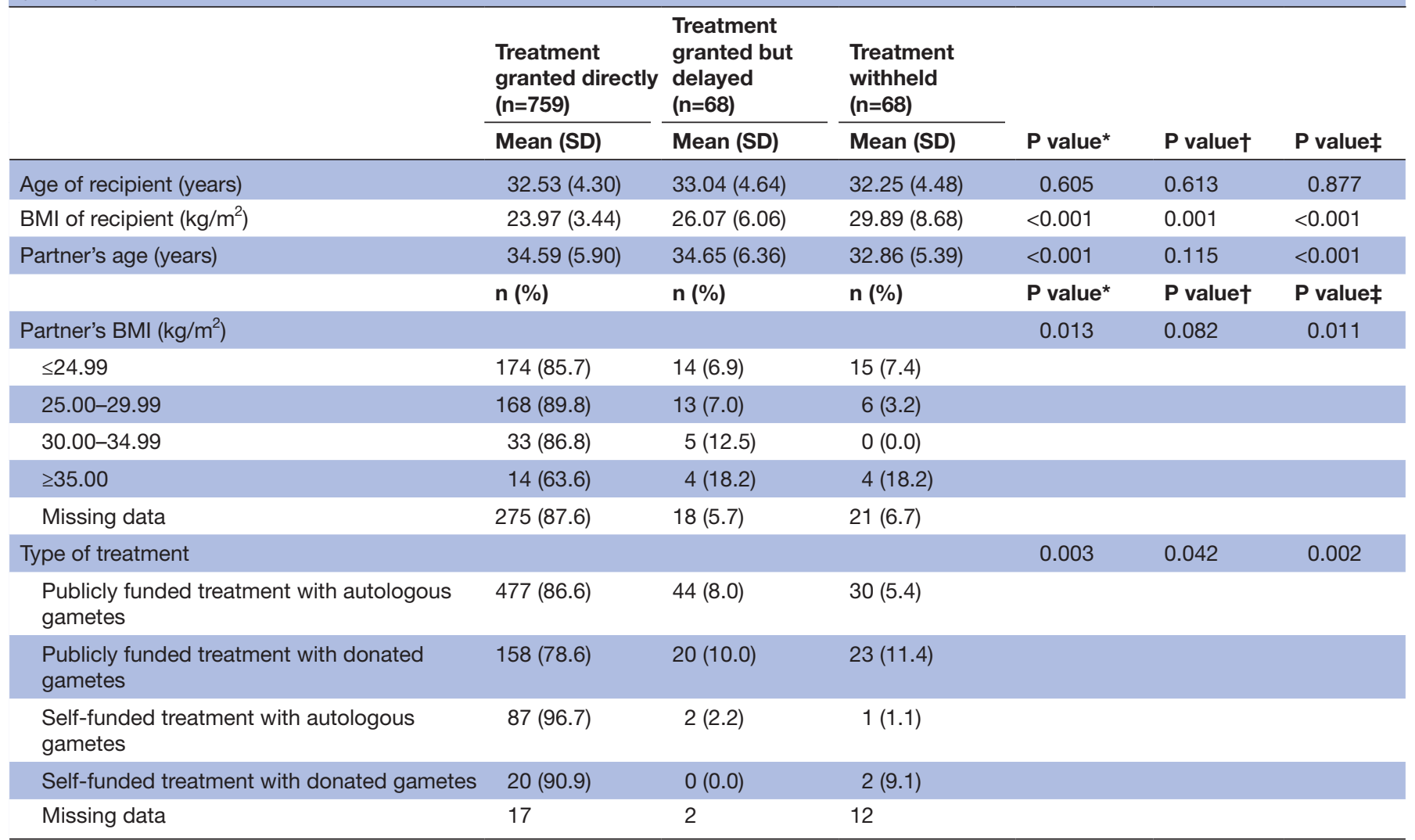

*ANOVA analysis across all groups.

†Comparison between granted and delayed treatments.

$\ddagger$ Comparison between granted and withheld treatments.

ANOVA, analysis of variance; ART, assisted reproductive technologies; BMI, body mass index.

the condition in question. Other reasons for delaying the granting of treatment were financial challenges, substance abuse, smoking and social reasons (table 3). Only after the necessary adjustments were made (such as change of employment status, and so on) were the candidates accepted for treatment. The main reasons for withholding treatment completely were primarily increased BMI, followed by somatic illness and mental disorders. Of those denied treatment, $13.5 \%$ of cases were due to insufficient self-reported time attempting a pregnancy (ie, shorter than 12 months) (table 4).

\section{DISCUSSION}

In this study, we have demonstrated that the great majority $(92 \%)$ of those seeking help for involuntary childlessness in two public fertility clinics in Sweden were granted treatment, either directly or after further evaluation. However, single relationship status and seeking treatment with donated gametes were associated with a higher probability of being denied treatment. There were no age differences between those granted or refused treatment; however, the mean BMI, both of the recipient and the partner (if any), was, overall, higher in the group where treatment was withheld.
The results show that candidates for self-funded treatment with autologous gametes have the highest rate of acceptance for treatment without additional assessment or delay $(96.7 \%)$; those candidates are not required to be assessed in accordance with the NBHW assessment guidelines, ${ }^{17}$ nor do they need to fulfil the eligibility criteria for publicly funded treatment. On the other hand, candidates for publicly funded treatment with donated gametes have the lowest rate of direct acceptance for treatment (78.6\%) since there are additional standards to meet. Interestingly, the proportion of direct acceptance is higher among heterosexual couples seeking treatment with donated gametes versus (female) same-sex couples and single women; it is thus open for question whether prejudice concerning relationship status and sexual orientation or other possible reasons could partly account for the differences in treatment access beyond the established NBHW criteria. In addition, all candidates are assessed regarding their willingness to disclose to the child the donor origin of the gametes employed for his/her conception; the unwillingness thus can be regarded as one more justified reason to delay or decline treatment. Furthermore, it is surprising that many clinics, including the two clinics at which this study was conducted, require for candidates to meet similar standards in order to be eligible for ART 
Table 2 Outcome (granted/delayed/denied ART treatment) reported by relationship status and gamete origin

\begin{tabular}{|c|c|c|c|}
\hline & $\begin{array}{l}\text { Donated } \\
\text { gametes }\end{array}$ & $\begin{array}{l}\text { Autologous } \\
\text { gametes }\end{array}$ & \\
\hline & n (\%) & n (\%) & $P$ value \\
\hline Single & & & N/A \\
\hline Granted & $92(73.0)$ & 0 & \\
\hline Delayed & $14(11.1)$ & 0 & \\
\hline Denied & $20(15.9)$ & 0 & \\
\hline Heterosexual $^{*}$ & & & 0.832 \\
\hline Granted & $29(85.3)$ & $564(88.0)$ & \\
\hline Delayed & $3(8.8)$ & $46(7.2)$ & \\
\hline Denied & $2(5.9)$ & $31(4.8)$ & \\
\hline Same sex & & & $\mathrm{N} / \mathrm{A}$ \\
\hline Granted & $63(88.7)$ & 0 & \\
\hline Delayed & $3(4.2)$ & 0 & \\
\hline Denied & $5(7.1)$ & 0 & \\
\hline \multicolumn{4}{|c|}{ Combined gamete origin } \\
\hline Accepted & $184(79.6)$ & $564(88.0)$ & $<0.001$ \\
\hline Delayed & $20(8.7)$ & $46(7.2)$ & \\
\hline Denied & $27(11.7)$ & $31(4.8)$ & \\
\hline
\end{tabular}

*Twenty heterosexual cases with missing data regarding gamete origin.

ART, assisted reproductive technologies; N/A, not applicable.

treatment of any kind. ${ }^{19}$ This means that the majority-if not all—of the ART candidates undergo some kind of evaluation of their psychological and social circumstances, regardless of whether their treatment will entail the use of donor gametes or not. More specifically, despite the absence of established and legally mandatory application of the NBHW criteria in the heterosexual couples group treated with autologous gametes, $12 \%$ of them were either denied treatment or had it delayed on similar grounds as when donated gametes were employed. This indicates the fact that fertility specialists consider psychosocial and medical assessments important in

Table 3 Reasons behind a treatment being delayed but granted after further evaluation

\begin{tabular}{lc}
\hline & $\mathbf{n}=64$ \\
\cline { 2 - 2 } & $\mathbf{n}(\%)^{*}$ \\
\hline Excess body weight & $11(17.2)$ \\
\hline Psychiatric disorder & $21(32.8)$ \\
\hline Physical disorder & $7(10.9)$ \\
\hline Social reasons & $4(6.3)$ \\
Substance abuse & $6(9.4)$ \\
Smoking/tobacco use & $3(4.7)$ \\
Advanced age (recipient) and relationship problem & $2(3.1)$ \\
\hline Financial reasons/occupational constraints & $6(9.4)$ \\
\hline Other & $4(6.3)$ \\
\hline
\end{tabular}

*Four cases with missing data.
Table 4 Reasons behind a treatment being withheld

\begin{tabular}{ll}
\hline & $\mathbf{n}=59$ \\
\cline { 2 - 2 } & $\mathbf{n}(\%)^{*}$ \\
\hline Excess body weight & $20(33.9)$ \\
\hline Psychiatric disorder & $6(10.2)$ \\
Physical disorder & $8(13.5)$ \\
Social reasons & $3(5.1)$ \\
Substance abuse & $1(1.7)$ \\
Smoking/tobacco use & $2(3.4)$ \\
Advanced age (recipient) and relationship problem & $2(3.4)$ \\
Financial reasons/occupational constraints & $3(5.1)$ \\
Incomplete ongoing evaluation & $5(8.5)$ \\
Insufficient time attempting pregnancy & $8(13.5)$ \\
Not eligible for publicly subsidised treatment (not & $1(1.7)$ \\
childless) & \\
\hline
\end{tabular}

${ }^{*}$ Nine cases with missing data.

all kinds of ART treatment. In fact, an analysis of focus group discussions with clinic staff at four public fertility clinics in Sweden by Lind ${ }^{19}$ showed that a majority of clinic staff argue in favour of the consideration of parenting adequacy of all candidates. They also ascribe to themselves responsibility towards society and taxpayers for the parenting evaluations they perform and the treatments they consent to provide. ${ }^{19}$ This could be attributed to the public character of the healthcare system in Sweden as well as the state's role in ensuring the health and well-being of children that result from a public treatment. But although one would expect that the 'restrictive policy' regulating in vitro fertilisation (IVF) access in Sweden serves financial interests, the degree of utilisation of IVF services in the country is nevertheless among the highest in Europe. ${ }^{20}$

In our study, obesity was among the major reasons for ART treatment being delayed (17\%) or completely withheld $(34 \%)$. This finding is not surprising since there is accumulating evidence that weight gain and obesity throughout adulthood have harmful effects both on future fertility and the time needed to achieve a pregnancy, as well as on the risk of pregnancy loss among obese women. ${ }^{21}$ Obesity, especially maternal morbid obesity (ie, BMI $\geq 40 \mathrm{~kg} / \mathrm{m}^{2}$ ), is associated with unfavourable obstetric outcomes such as pre-eclampsia, shoulder dystocia, stillbirth, ${ }^{22}$ as well as adverse neonatal outcomes. ${ }^{23}$ In addition to the long-term consequences of pregnancy safety, concerns have also been expressed regarding the efficacy, cancellation rate and cost-effectiveness of IVF treatment, as well as the short-term safety of obese women during IVF procedures. ${ }^{24}$ However, one can question whether the delay due to the weight loss attempt has in fact a beneficial or instead a counteractive effect on the chances of pregnancy, taking also into account the detrimental effect of time and female ageing on ovarian reserve, especially among women above 35 years of age.$^{25}$ Moreover, the imposed upper cut-off limit for providing ART treatment may vary widely among different countries and different institutions. ${ }^{24}{ }^{26}$ In 
fact, certain IVF centres in the USA may provide treatment for women with BMI as high as $62 \mathrm{~kg} / \mathrm{m}^{2} .{ }^{26}$ In the two institutions included in the current study, the provider policy was stricter, with a cut-off limit lying below 30 or $35 \mathrm{~kg} / \mathrm{m}^{2}$, respectively.

The major strength of our study is its two-centre design, comprising clinics that both perform publicly subsidised as well as self-funded IVF treatments with both autologous and donated gametes, and hence recruit a wide range of individuals of varying socioeconomic backgrounds from both urban and rural areas. Furthermore, the study performed is one of the largest on the subject published thus far. In order to address the research question, a valid instrument, based on the recommendations of an official authority (ie, NBHW), was developed and employed. Another strength is that the study protocol enquired both about cases where candidates were denied treatment and about cases where the clinics hesitated to grant treatment without additional evaluation. Finally, since the evaluation of candidates treated with thirdparty gametes in private settings in Sweden will be regulated by the same mandatory by law assessment guidelines presented in the current study, we anticipate that the study findings will be in large admissible even there with satisfactory generalisability.

The current study has however a number of limitations. No information regarding the type of donated gamete (ie, sperm or egg) among heterosexual couples was recorded. In addition, the quantitative data of this study reveal little about the deliberations and standpoints of the clinicians in Sweden regarding these ethically challenging questions and whether these might affect their decisions. How prone one is to bend the rules might differ globally and may be subject to cultural differences. Findings from an Israeli study on physicians' attitudes revealed that $90 \%$ of physicians interviewed in Israel declared that they would not deny treatment to candidates affected by chronic disease or low socioeconomic status, those who have minor mental health problems, those with 'light' criminal records (charged with felonies of up to 3 years of imprisonment), those who occasionally consume drugs, or those who, as a couple, have had problems in their relationship. ${ }^{27}$ These findings could, however, be explained by the widely supported pronatalist approach of the Israeli state. No similar study has been performed in Sweden thus far. In addition, a small number of couples/singles dropped out of the evaluation process by choice before conclusion of the assessment. It is uncertain what the result of the fertility assessment would have been and whether the dropout rate would reflect a higher proportion of treatment refusal had the individuals been evaluated. Furthermore, one group of individuals was denied treatment due to insufficient time attempting pregnancy or due to secondary infertility (ie, already having joint custody of a child). Therefore, one could argue that the findings could be associated with the publicly financed character of the health system in Sweden and it is open to question whether the outcome would be different if ART treatments were exclusively privately funded.

While there is no common plan established regarding the requirements for ART access, especially in treatments subsidised by the state, certain similarities seem to exist between countries. In fact, a comparative analysis by the European Union (EU) revealed that most EU member states offered at least partial financial coverage for IVF treatments with limitations of access in public institutes pertaining to female age, sexual orientation and marital status, ${ }^{28}$ limitations also implemented in Sweden. Furthermore, the eligibility criteria imposed have often been chosen on the same grounds as in Sweden, that is, focusing on the welfare of future individuals (ie, preconception welfare principle).$^{29}$ Lastly, the reasons for treatment refusal presented in the study have been previously reported in the literature and do not relate to conditions met exclusively in Sweden. ${ }^{1-7}$ These similarities increase the applicability of the findings even to public settings outside of Scandinavia.

\section{CONCLUSION}

By performing the present study, we attempted to understand the current standard of care regarding reproductive evaluation and treatment in a public setting in Sweden. The great majority $(>90 \%)$ of those undergoing fertility evaluation were granted treatment after medical and psychosocial assessment. For single women, the percentage of candidates who were accepted for treatment without additional in-depth assessment was lower (73\%). All single women candidates, as well as couple candidates for donor gamete treatment, must undergo the psychological and social assessments prescribed by Swedish legislation in third-party reproduction. In addition, the requirements regarding single candidates' social network are more comprehensive than in relation to couple candidates. However, the fact that several candidates for treatment with autologous gametes were denied treatment on socioeconomical or psychological grounds, despite the fact that Swedish legislation prescribes that such an assessment is required only when gametes from a donor are used, suggests that clinicians regard the assessment of all candidates' psychological and social circumstances as important. Thus, the need for clear and comprehensive policy guidelines is emphasised, especially in cases where the treating physician lacks experience. These policy guidelines should regulate the provision of treatment in an objective and evidence-based manner.

\section{Author affiliations}

${ }^{1}$ Department of Women's and Children's Health, Uppsala University, Uppsala, Sweden

2Department of Clinical Sciences, Obstetrics and Gynecology, Lund University, Lund, Sweden

${ }^{3}$ Department of Thematic Studies-Child Studies, Linköping University, Linköping, Sweden

${ }^{4}$ Obstetrics and Gynaecology, Department of Clinical and Experimental Medicine, University Hospital in Linköping, Linköping, Sweden

Acknowledgements We thank Annica Dahl and the medical personnel in the participating clinics for the rigorous data collection, as well as Marie Bladh for her contribution to the statistical analysis.

Contributors All authors (EE, JL, ASS, PL, GS) have equally contributed to the design of the study, data collection, interpretation of the results, drafting of the manuscript, as well as critical revision of the final version submitted. 
Funding The study was supported by FORTE, the Swedish Research Council for Health, Working Life and Welfare (grant number 2016-00583). EE has a parttime research position funded by Uppsala University Hospital (grant number ALF 1040530/2019).

Competing interests EE has received lecture fee from Gideon Richter outside the submitted work.

Patient consent for publication Not required.

Ethics approval The study was approved by the 'Regional Ethical Review Board in Linköping, Sweden', part of the Swedish Ethical Review Authority (Dnr 2017/185$31)$. Neither written nor oral informed consent was gathered from the individuals seeking treatment as all data registered were anonymised.

Provenance and peer review Not commissioned; externally peer reviewed.

Data availability statement Data are available upon reasonable request. The data sets generated and analysed during the current study are not publicly available and cannot be uploaded at any website due to the risk of compromising the individual privacy of participants. On the other hand, the data are available from the responsible department upon reasonable request. Any interested parties are welcome to contact the authors, who will then fill out the agreements necessary when sharing data and after approval by the Regional Ethics Board in Linköping, Sweden. All data regarding the current study are available on request to the Department of Women's and Children's Health, Uppsala University; kbh@kbh.uu.se.

Open access This is an open access article distributed in accordance with the Creative Commons Attribution Non Commercial (CC BY-NC 4.0) license, which permits others to distribute, remix, adapt, build upon this work non-commercially, and license their derivative works on different terms, provided the original work is properly cited, appropriate credit is given, any changes made indicated, and the use is non-commercial. See: http://creativecommons.org/licenses/by-nc/4.0/.

\section{ORCID iD}

Evangelia Elenis http://orcid.org/0000-0002-2253-4682

\section{REFERENCES}

1 Siegel AM, Ravitsky V. Women with mental illness seeking assisted reproduction considerations in ethical candidate selection. Curr Psychiatry Rep 2018;20:70.

2 Golberstein E, Gonzales G, Meara E. How do economic downturns affect the mental health of children? Evidence from the National health interview survey. Health Econ 2019;28:955-70.

3 Thompson K, McDougall R. Restricting access to ART on the basis of criminal record : an ethical analysis of a state-enforced "presumption against treatment" with regard to assisted reproductive technologies. J Bioeth Inq 2015;12:511-20. doi:10.1007/s11673015-9622-z

4 Tonkens R. Infertilitism: unjustified discrimination of assisted reproduction patients. Monash Bioeth Rev 2018;35:36-49.

5 Dondorp W, de Wert G, Pennings G, et al. Lifestyle-related factors and access to medically assisted reproduction. Hum Reprod 2010;25:578-83. doi:10.1093/humrep/dep458

6 Gillett WR, Peek JC, Herbison GP. Development of clinical priority access criteria for assisted reproduction and its evaluation on 1386 infertile couples in New Zealand. Hum Reprod 2012;27:131-41.

7 Brown $\mathrm{RCH}$. Irresponsibly infertile? obesity, efficiency, and exclusion from treatment. Health Care Anal 2019;27:61-76.
8 Ministry of Health and Social Affairs. Directive on the use of tissues and cells in healthcare and clinical research (2009:32) Sect. 7,8,9,19, 2009.

9 De Wert G, Dondorp W, Shenfield F, et al. ESHRE Task force on ethics and law 23: medically assisted reproduction in singles, lesbian and gay couples, and transsexual people. Hum Reprod 2014;29:1859-65.

10 Ethics Committee of American Society for Reproductive Medicine. Access to fertility treatment by gays, lesbians, and unmarried persons: a committee opinion. Fertil Steril 2013;100:1524-7.

11 Stern JE, Cramer CP, Garrod A, et al. Access to services at assisted reproductive technology clinics: a survey of policies and practices. Am J Obstet Gynecol 2001;184:591-7.

12 Thorpe R, Croy S, Petersen K, et al. In the best interests of the child? regulating assisted reproductive technologies and the wellbeing of offspring in three Australian states. Int $J$ Law Policy Family 2012;26:259-77.

13 Lee E, Macvarish J, Sheldon S. Assessing child welfare under the human fertilisation and embryology act 2008: a case study in medicalisation? Sociol Health IIIn 2014;36:500-15.

14 Gurmankin A, Caplan A, Braverman A. Screening practices and beliefs of assisted reproductive technology programs. Fertil Steril 2005;83:61-7.

15 Ministry of Health and Social Affairs. The genetic integrity act (2006:351). Sect. 5, 2006.

16 Lind J. The rights of intended children: the best interests of the child argument in assisted reproduction policy. Childhood 2019;26:352-68.

17 National Board of Health and Welfare. The National Board of Health and Welfare's Knowledge Support: Assisted Reproduction with donated gametes. Stockholm, Sweden: Socialstyrelsen, 2016.

18 Swedish Association of Local Authorities and Regions. Recommendation: assisted reproduction, 2016.

19 Lind J. Child welfare assessments and the regulation of access to publicly funded fertility treatment. Reprod Biomed Soc Online 2020;10:19-27.

20 Berg Brigham K, Cadier B, Chevreul K. The diversity of regulation and public financing of IVF in Europe and its impact on utilization. Hum Reprod 2013;28:666-75.

21 Gaskins AJ. Recent advances in understanding the relationship between long- and short-term weight change and fertility. F1000Res 2018;7:1702.

22 Cedergren MI. Maternal morbid obesity and the risk of adverse pregnancy outcome. Obstet Gynecol 2004;103:219-24.

23 Blomberg M, obesity M. Maternal obesity, mode of delivery, and neonatal outcome. Obstet Gynecol 2013;122:50-5.

24 Rittenberg V, Seshadri S, Sunkara SK, et al. Effect of body mass index on IVF treatment outcome: an updated systematic review and meta-analysis. Reprod Biomed Online 2011;23:421-39.

25 Tremellen K, Wilkinson D, Savulescu J. Should obese women's access to assisted fertility treatment be limited? A scientific and ethical analysis. Aust N Z J Obstet Gynaecol 2017;57:569-74.

26 Kaye L, Sueldo C, Engmann L, et al. Survey assessing obesity policies for assisted reproductive technology in the United States. Fertil Steril 2016;105:703-6.

27 Sperling D, Simon Y. Attitudes and policies regarding access to fertility care and assisted reproductive technologies in Israel. Reprod Biomed Online 2010;21:854-61.

28 ESHRE. Comparative analysis of medically assisted reproduction in the EU: regulation and technologies. , 2008: 32, 1-166.

29 HFEA. The UK Human Fertilisation and Embryology Act: Sect (13)5. In: Reformed 2008 by the human fertilisation and embryology authority (HFEA), 1990. 